\title{
Como a Gestão de Identidade Federada pode contribuir com a Proteção de Dados Pessoais?
}

\author{
Michelle Silva Wangham ${ }^{1,2}$ \\ ${ }^{1}$ Laboratório de Sistemas Embarcados e Distribuídos (LEDS) \\ Universidade do Vale do Itajaí (UNIVALI) - Itajaí, SC - Brasil \\ ${ }^{2}$ Rede Nacional de Ensino e Pesquisa (RNP) \\ wanghameunivali.br
}

\begin{abstract}
Resumo. O principal objetivo da Lei Geral de Proteção de Dados (LGPD) é a privacidade e o foco da Lei está em como as organizações processam, armazenam e protegem dados pessoais. Os sistemas de gestão de identidades, por armazenarem, manipularem e protegerem os dados pessoais, precisam estar em conformidade com a LGPD. O modelo de gestão de identidade adotado na Comunidade Acadêmica Federada (CAFe) possui características que contribuem com a proteção de dados pessoais. O objetivo desta palestra curta é esclarecer como a proteção de dados pessoais deve ser tratada nos provedores de identidades e de serviços da CAFe e como esta proteção pode ser reforçada com boas práticas de segurança, controles de autenticação mais robusto e políticas de privacidade.
\end{abstract}

\section{Proposta}

A Lei n ${ }^{0}$ 13.709/2018 - Lei Geral de Proteção de Dados Pessoais (LGPD) [Brasil 2018], sancionada em agosto de 2018, dispõe sobre o tratamento de dados pessoais, inclusive nos meios digitais, por pessoa natural ou por pessoa jurídica de direito público ou privado. O principal foco da LGPD é a privacidade e esta determina a adoção de medidas de segurança para evitar qualquer forma de utilização inadequada dessas informações pessoais e sigilosas. Os sistemas de gestão de identidades (GId), por armazenarem, manipularem e protegerem os dados pessoais, precisam estar em conformidade com a LGPD.

O modelo de identidade federada adotado na Comunidade Acadêmica Federada (CAFe) possui características que contribuem com a proteção de dados pessoais, tais como: a gestão de contas de usuários sendo provida pela instituição de origem (home) e não pelos serviços da federação, o uso de termos de consentimento para liberação de atributos de identidade e as políticas de uso. Para garantir a segurança e privacidade, a transformação digital no ensino e na pesquisa deve envolver a CAFe e outros controles e boas práticas de segurança. Vale ainda destacar que as Instituições de Ensino e Pesquisa precisarão estar em conformidade com a Lei Geral de Proteção de Dados até agosto de 2020 .

O objetivo desta palestra curta é esclarecer como a proteção de dados pessoais deve ser tratada nos provedores de identidades e de serviços da CAFe e como esta proteção pode ser reforçada com boas práticas de segurança, controles de autenticação mais robusto (Multi-factor Authentication -MFA) e políticas de privacidade. Dentre o conjunto de boas práticas de segurança, serão mencionadas as medidas preventivas para proteger a 
organização de um ataque e as medidas a serem adotadas em incidentes, conforme definido no SIRTFI (Security Incident Response Trust Framework for Federated Identity) ${ }^{1}$ da REFEDS (Research and Education FEDerations).

Devido ao regulamento geral sobre proteção de dados pessoais Europeia, a General Data Protection Regulation - GDPR ${ }^{2}$, as federações acadêmicas Europeias já possuem um conjunto de controles e boas práticas que visam contribuir com a proteção de dados pessoais. Destaca-se, por exemplo, o Código de Conduta de Proteção de Dados (Data protection Code of Conduct - $\mathrm{CoCo})^{3}$, elaborado pela Gèant para atender aos requisitos da GDPR no contexto de autenticação federada de usuários e liberação de seus atributos. O CoCo oferece instruções normativas, não normativas (informais), ferramentas de monitoramento e um conjunto de orientações (receitas) elaboradas para Provedores de Serviços (SPs) ${ }^{4}$, Instituições Home (e seus Provedores de Identidades - IdPs) ${ }^{5}$ e Operadores de Federações ${ }^{6}$ que habilita a liberação segura de atributos entre IdPs e SPs na União Europeia. Dentre as instruções normativas, destacam-se o código de conduta para SPs, um especificação ${ }^{7}$ que define um atributo SAML 2.0 Entity Category para SPs que se declaram em conformidade com o código de conduta e um para IdPs, que desejam inte-

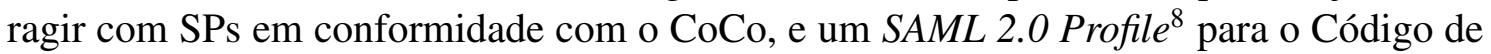
Conduta de Proteção de Dados. Pretende-se nesta palestra trazer estas experiências internacionais e quais os desafios para adoção destes controles e boas práticas nas instituições brasileiras.

Como mencionado, a palestra visa discutir aspectos de segurança e privacidade para que as instituições que já aderiram à CAFe entendam seus desafios e comecem a se adequar a LGPD. Por fim, pretende-se apresentar sugestões de como a RNP, entidade responsável pela operação da $\mathrm{CAFe}$, pode contribuir com a conformidade dos membros da CAFe à LGPD.

\section{Referências}

Brasil (2018). Lei geral de proteção de dados pessoais (lgpd). http: //www.planalto.gov.br/ccivil_03/_ato2015-2018/2018/lei/ L13709compilado.htm.

\footnotetext{
${ }^{1}$ https://refeds.org/wp-content/uploads/2016/01/Sirtfi-1.0.pdf

${ }^{2}$ https://ec.europa.eu/commission/priorities/justice-and-fundamental-rights/ data-protection/2018-reform-eu-data-protection-rules_en

${ }^{3}$ https://wiki.refeds.org/display/CODE/Data+Protection+Code+of+ Conduct + Home

${ }^{4}$ https://wiki.geant.org/display/eduGAIN/Recipe+for+a+Service+Provider

5 https://wiki.geant.org/display/eduGAIN/CoCo+Recipe+for+a+Home+ Organisation

${ }^{6}$ https://wiki.geant.org/display/eduGAIN/Recipe+for+a+Federation+ Operator

${ }^{7}$ https://wiki.refeds.org/display/CODE/Entity+Category+Definition \\%3A+ Data+protection+Code+of+Conduct

${ }^{8}$ https://wiki.refeds.org/display/CODE/SAML+2+Profile+for+the+Data+ Protection+Code+of+Conduct
} 